\title{
Problems with the pathological diagnosis of osteonecrosis
}

\author{
Shobha Parajuli ${ }^{1}$ - John R. Fowler ${ }^{2} \cdot$ Easwaran Balasubramanian $^{3}$. \\ William R. Reinus $^{4}$ • John P. Gaughan ${ }^{5}$ • Daniel I. Rosenthal ${ }^{6}$. Jasvir S. Khurana ${ }^{1}$
}

Received: 14 October 2013 /Revised: 28 September 2015 / Accepted: 12 October 2015 / Published online: 22 October 2015

(C) ISS 2015

Establishing the pathological diagnosis of osteonecrosis of the femoral head $(\mathrm{ONFH})$ has historically been given little importance. In fact, even the need to histologically examine femoral heads (that have been excised during routine hip arthroplasty) has been debated $[1,2]$. Perhaps this lack of interest is because ONFH becomes significant only when it leads to secondary degenerative joint disease (DJD), and because the treatment of hip ONFH and DJD is the same. This leads to a lack of interest by the surgeon in the pathological diagnosis of femoral heads, because the diagnosis does not alter the subsequent management or outcome.

However, a few years ago, one of the authors was part of an advisory committee meeting at the FDA where a group of drugs was being investigated for a possible side effect of ONFH [3]. Although the final adjudication was based upon multiple factors, one problem that came to light during the discussion was the relative uncertainty in the baseline preva-

Jasvir S. Khurana

jkhurana@temple.edu

1 Department of Pathology, Temple University Hospital, 3401 North Broad Street, Philadelphia, PA 19104, USA

2 Department of Orthopaedics, University of Pittsburgh, Suite 911, Kaufmann Bldg 3471 Fifth Avenue, Pittsburgh, PA 15213, USA

3 Temple University Hospital, 3401 North Broad Street, Philadelphia, PA 19104, USA

4 Department of Radiology, Temple University Hospital, 3401 North Broad Street, Philadelphia, PA 19104, USA

5 Biostatistics Consulting Center, Temple University School of Medicine, 3420 North Broad Street, Philadelphia, PA 19140, USA

6 Department of Radiology, Massachusetts General Hospital, 175 Cambridge Street, Boston, MA 02114, USA lence rates of ONFH. It became obvious that although the diagnosis of osteonecrosis may not matter to the individual patient or surgeon, it becomes important in developing prevalence data upon which future drug trials may perhaps depend.

Literature on the prevalence of ONFH is surprisingly sparse. A literature review (Medline, 1946 to present), using the key words prevalence and osteonecrosis or avascular necrosis yielded 272 articles; however, it turned out that most of these did not deal with ONFH in general patient populations. Instead, the vast majority of articles deal with specific patients such as those with kidney disease, collagen vascular diseases, immunosuppression, genetic conditions, transplantation or hematological problems amongst whom the prevalence of $\mathrm{ONFH}$ is expected to be higher than in the general public. It is clear that the prevalence of osteonecrosis depends on the patient mix [4]. After excluding articles that were limited to certain patient groups or diseases, there were only a handful of articles that dealt with general patient populations. These articles generally suggested that in the United States, ONFH affects about $10 \%$ of all hips that are replaced [5-8]; however, some of these articles were not very clear about how the diagnoses were made (radiologically or pathologically).

In some countries, for example, Japan and South Korea, there has been an attempt to study the prevalence of ONFH at a national level [9-11]. In Japan, a questionnaire was sent to orthopedic departments, and in South Korea, insurance data were used. Both methods have the benefit of including patients who may not present for surgery, but the disadvantage that the use of a code for the diagnosis does not reveal whether the diagnosis was only clinically suspected or definitively made, or how it was made: radiologically versus pathologically. Studies from Asia also reveal a higher prevalence-as many as 50-60\% of arthroplasties are performed to treat ONFH $[10,12]$. However, this may be the result of a lower 
prevalence of osteoarthritis rather than an increased prevalence of ON.

There are several problems involved in studying the prevalence of ONFH. First, the term prevalence is used by biostatisticians as a measure of the presence of a condition in a particular population. For ONFH this is not known because we only know of the presence of ONFH in the femoral heads of patients who present for surgery and not in the general population. It is possible that some patients with ONFH do not have surgery, perhaps because their symptoms are insufficiently severe or because of other comorbidities that might preclude surgery.

Another problem is encountered, because the natural history of osteonecrosis is to heal. We know from longitudinal radiological and histological studies of patients with various osteochondritidies and other forms of osteonecrosis that necrotic bone has the capacity to heal, and that this may occur with or without secondary osteoarthritis. The cases that come to surgery, therefore, are those that collapse and secondary DJD occurs before the healing. We do not know the numbers of people in whom the reverse occurs (i.e., where healing is completed before collapse). It appears plausible that the smaller the region of osteonecrosis, the more likely it is to heal. Therefore, it is quite likely that less severely affected individuals may not present for surgery $[13,14]$.

Ideally, therefore, the diagnosis of ONFH should be made by some non-invasive means and ideally this would have a perfect correlation with the gold standard, the pathological examination. This would also ideally be combined with a long-term follow-up of the population; however, we clearly have no such ideal modalities and it is not possible to subject the entire population to radiological studies at frequent intervals.

A third problem is that the available imaging modalities (X-ray, nuclear scans, MRI) have differing sensitivities and specificities and their interpretation may be subject to some inter- and intra-observer differences [15-20]. Interestingly, in a recent study of radiological practice published in this journal, the authors also concluded that current radiological practice in diagnosing ONFH was non-uniform and unsatisfactory [21]. With new modalities being brought into practice, it becomes difficult to compare data across different time periods.

The problems with the pathological diagnosis also bear some scrutiny. This is supposed to be the "gold standard" by which other modalities are judged; however, problems in the pathological diagnosis have not been well explored. For example, unlike the radiology literature, where there are a number of studies on the reliability and precision of femoral head osteonecrosis [22, 23], we are not aware of any studies that have looked at intra- and inter-observer variability in the pathological diagnosis of osteonecrosis. Although osteonecrosis seems to be a fairly straightforward diagnosis, in the location of the femoral head there are no established quantitative criteria. This is important, because a few necrotic trabeculae often accompany osteoarthritis, but these should not be diagnosed as ONFH, as the size of the focus is not clinically significant $[24,25]$. Usually, clinically significant ONFH is accompanied by bone marrow necrosis and signs of repair such as creeping substitution. Interestingly, it has also been postulated that in some cases, rapidly progressive osteoarthritis may secondarily cause osteonecrosis [26]. If this is true, then in these cases bone necrosis is an effect, not a cause, of the arthritis. It is therefore possible that the pathological diagnosis of ONFH suffers from a lack of standardization.

An additional problem that is inherent in pathology is that the correctness and frequency of a diagnosis often depends upon the intensity with which it is sought. In other words, if you don't think of it, you won't look for it and you won't find it. A careful search for a focus of osteonecrosis ideally requires the femoral head to be serially sectioned and multiple suspicious areas to be examined. However, for femoral heads (as in other cases that are sent to the laboratory for documentation alone), there could be a tendency toward less careful sectioning (with the feeling that the diagnosis may not really matter). This could perhaps be compounded by the lack of interest in the surgical team. Pathologists often receive specimens for documentation alone and once documentation is over, the job is considered done. For example, an appendix that is removed incidentally might undergo one histological section, whereas if the appendix was removed for appendicitis it might undergo more (or even be totally sampled) to document the presence or absence of the clinical diagnosis.

This behavioral pattern is not limited to pathologists alone. All clinical specialties demonstrate the alteration in the intensity of looking for a diagnosis depending upon the clinical suspicion. For example, if a teenager comes to a doctor's office stating that he or she is there because their parents told them to come for a check-up, they might undergo a physical examination, perhaps a blood test, but not much more. On the other hand, if the history provided is that of ankle swelling, fever, and pain, they might undergo a more involved work-up including X-rays and a more detailed blood test. Thus, the history provided matters in the intensity and direction of our work-up.

If this hypothesis is true, then femoral heads removed for degenerative joint disease might undergo less sectioning

Table 1 Blocks submitted for evaluation

\begin{tabular}{lllll}
\hline $\begin{array}{l}\text { Diagnosis on pathology } \\
\text { requisition forms }\end{array}$ & $n$ & $\begin{array}{l}\text { Mean number } \\
\text { of blocks (SD) }\end{array}$ & Minimum & Maximum \\
\hline Osteonecrosis & 31 & $6.06(7.22)$ & 2 & 36 \\
No osteonecrosis & 358 & $2.18(1.29)$ & 1 & 17 \\
\hline
\end{tabular}

$S D$ standard deviation 
Table 2 Blocks submitted for evaluation

\begin{tabular}{|c|c|c|c|c|c|}
\hline Diagnosis on requisition form & Pathological diagnosis (pathology report) & $n$ & Mean number of blocks (SD) & Minimum & Maximum \\
\hline Osteonecrosis & No osteonecrosis & 9 & $11.00(11.90)$ & 2 & 36 \\
\hline No osteonecrosis & & 328 & $2.16(1.25)$ & 1 & 17 \\
\hline Osteonecrosis & Osteonecrosis & 22 & $4.04(2.39)$ & 2 & 11 \\
\hline No osteonecrosis & & 30 & $2.40(1.69)$ & 1 & 8 \\
\hline
\end{tabular}

(perhaps one section from the articular cartilage to document osteoarthritis), but if removed for osteonecrosis they might undergo more sectioning to look for the lesion in question (and perhaps many more sections if the pathology does not correlate with the clinical impression). More importantly, if the history provided is incorrect or misleading, this might lead to missing the focus of osteonecrosis and perhaps an alternative diagnosis of degenerative joint disease (which invariably accompanies ONFH, especially in hips that present for arthroplasty). Again, perhaps this is not important in the individual case, but may significantly alter the statistics of the prevalence of ONFH.

In order to test this hypothesis we retrospectively reviewed (after IRB permission) the pathological diagnoses (pathology reports), pathology requisition forms (pathology request forms), radiological reports, and medical records of all the patients at our institution who underwent excision of at least one femoral head between January 2007 and December 2011. We found 389 femoral heads from arthroplasties performed on 357 patients. We found that the cases were fairly evenly distributed amongst our pathologists, with no discernible clustering around any specific pathologist. We were interested in the pathological diagnosis and specifically in whether previous knowledge of the clinical and/or radiological diagnosis influenced the extent of tissue sampling. We therefore reviewed the pathology requisition/request forms for information that had been provided to the assigned pathologist at the time of submission. We considered the possibility that the diagnosis that is entered on the pathology requisition/request forms might differ from the real "clinical diagnosis" made by the surgeon, as the requisition form is often filled out by the operating room staff, who may sometimes have an incomplete understanding of the case. For example, let us think of an alcoholic deep-sea diver who is on steroids for asthma and who presents with bilateral hip pain to a surgeon. The surgeon requests imaging studies, which confirm a clinical suspicion of bilateral hip osteonecrosis. This then is the surgeon's clinical diagnosis and is documented as such in the clinical charts and medical records. Now the patient is taken to the operating room for an arthroplasty. The operating room nurse, who is filling out the pathology requisition form, writes "femoral head with DJD" because, in that hospital, most femoral heads are removed because of DJD, or because he or she fails to check the history with the surgeon. This becomes the diagnosis available to the pathologist (who is unaware of the actual surgeon's diagnosis in the medical record).

For the study, we counted the number of tissue blocks that were sampled in each case of femoral head arthroplasty and matched this number against whether or not a diagnosis of ONFH had been provided on the pathology requisition forms. We recorded the pathologist's diagnosis, but did not revise the pathological diagnoses (even if we felt they were incorrect) because of our purpose was to find behavioral differences related to the history and clinical information that was provided and not to determine the accuracy of the diagnosis. We found that if the requisition sheet gave a diagnosis of ONFH, then the mean number of paraffin blocks examined in these cases was 6.1 per case. Whereas, if the requisition sheet did not give any diagnosis at all, or gave a diagnosis other than $\mathrm{ONFH}$, then the pathologist sampled 2.2 blocks per case. If a
Table 3 Diagnosis of ONFH by subspecialty

\begin{tabular}{llll}
\hline & Prevalence of osteonecrosis & Percentage & Percentage (95\% CI) \\
\hline Pathologist (pathology report) & $52 / 389$ & 13.37 & $9.98-17.53$ \\
Surgeon (medical record) & $62 / 389$ & 15.94 & $12.22-20.43$ \\
Radiologist (radiology report) & $69 / 347$ & 19.88 & $15.47-24.17$ \\
\hline
\end{tabular}

The number of cases (denominator) in the last row differs because only 347 imaging reports were available to us. The remaining cases ( 31 cases) had studies that were reviewed by the surgeon and returned. These cases have a surgeon's diagnosis but no radiologist's diagnosis. The results were obtained by reviewing the pathology reports, radiology reports and the clinical/hospital medical records (for the actual surgeon's diagnosis). The pathology requisition sheets (which do not reflect the true clinical diagnosis) were not utilized for Table 3

CI confidence interval 
diagnosis of osteonecrosis was communicated to the pathologist (via the requisition sheet), but no osteonecrosis was present histologically, the mean number of blocks increased further to 11 blocks per femoral head (Tables 1 and 2). As our study spanned a period of 5 years and many different pathologists, the data are unlikely to be biased by an outlier pathologist. We therefore believe that that these data show that pathologists try to confirm a clinical diagnosis of ONFH if it is suggested.

These data support the hypothesis that sampling by the pathologist might be influenced by the clinical question that is asked. If other institutions follow similar practices, then current published baseline estimates of the prevalence of ONFH could be seriously flawed.

Interestingly, a review of the pathology and radiology reports together with the clinical charts revealed some differences in the prevalence of ONFH. Thus, despite using exactly the same patients, the prevalence differed according to the specialty that made the interpretation (Table 3 ).

In summary, the true prevalence of ONFH is important because of the emergence of new therapeutic drugs and antibodies that might have ONFH as a side effect. However, currently available data on the prevalence of ONFH could be seriously flawed because of several reasons highlighted above. The term prevalence is incorrectly used, asymptomatic ONFH confounds the numbers, the size and other pathological criteria of ONFH are not standardized, and there are no good studies on inter- and intra-observer diagnoses amongst pathologists. The diagnosis of ONFH varies among specialties and the literature fails to mention, which methods were used for the diagnosis. We therefore advocate caution before applying historical data on ONFH to the evaluation of drugs that may either cause or prevent osteonecrosis. There is a need for a consensus definition of the histological features of ONFH and a careful study to improve our understanding of its prevalence.

\section{Compliance with ethical standards}

\section{Source of funding None.}

Conflict of interest The authors declare that they have no conflict of interest,

\section{References}

1. Kocher MS, Erens G, Thornhill TS, Ready JE. Cost and effectiveness of routine pathological examination of operative specimens obtained during primary total hip and knee replacement in patients with osteoarthritis. J Bone Joint Surg Am Vol. 2000;82-A(11): 1531-5.

2. Clark CR, Bauer T. Routine pathological examination of operative specimens from primary total hip and total knee replacement: another look. J Bone Joint Surg Am Vol. 2000;82-A(11):1529-30.
3. FDA. 2012 Meeting Materials, Arthritis Advisory Committee. 2012.

4. Pavelka K. Osteonecrosis. Baillieres Best Pract Res Clin Rheumatol. 2000;14(2):399-414.

5. Mankin HJ. Nontraumatic necrosis of bone (osteonecrosis). N Engl J Med. 1992;326(22):1473-9.

6. Coventry MB, Beckenbaugh RD, Nolan DR, Ilstrup DM. 2,012 total hip arthroplasties. A study of postoperative course and early complications. J Bone Joint Surg Am. 1974;56(2):273-84.

7. Mont MA, Hungerford DS. Non-traumatic avascular necrosis of the femoral head. J Bone Joint Surg Am. 1995;77(3):459-74.

8. Israelite CL, Garino JP. Osteonecrosis of the hip. Semin Arthroplast. 2005;16:27-32.

9. Hirota Y. Idiopathic Osteonecrosis of the femoral head: nationwide epidemiologic studies in Japan. In: Urbaniak JR, Jones JP, editors. Osteonecrosis-etiology, diagnosis and treatment. Chicago: American Academy of Orthopaedic Surgeons; 1997.

10. Kang JS, Park S, Song JH, Jung YY, Cho MR, Rhyu KH. Prevalence of osteonecrosis of the femoral head: a nationwide epidemiologic analysis in Korea. J Arthroplasty. 2009;24(8):117883.

11. Fukushima W, Fujioka M, Kubo T, Tamakoshi A, Nagai M, Hirota Y. Nationwide epidemiologic survey of idiopathic osteonecrosis of the femoral head. Clin Orthop Relat Res. 2010;468(10):2715-24.

12. Parsons SJ, Steele N. Osteonecrosis of the femoral head: part 1aetiology, pathogenesis, investigation, classification. Curr Orthop. 2007;21:457-63.

13. Mont MA, Zywiel MG, Marker DR, McGrath MS, Delanois RE. The natural history of untreated asymptomatic osteonecrosis of the femoral head: a systematic literature review. J Bone Joint Surg Am. 2010;92(12):2165-70.

14. Mulliken BD, Renfrew DL, Brand RA, Whitten CG. The prevalence and natural history of early osteonecrosis $(\mathrm{ON})$ of the femoral head. Iowa Orthop J. 1994;14:115-9.

15. Mont MA, Jones LC, Hungerford DS. Nontraumatic osteonecrosis of the femoral head: ten years later. J Bone Joint Surg Am. 2006;88(5):1117-32.

16. Beltran J, Herman LJ, Burk JM, Zuelzer WA, Clark RN, Lucas JG, et al. Femoral head avascular necrosis: MR imaging with clinicalpathologic and radionuclide correlation. Radiology. 1988;166(1 Pt 1):215-20.

17. Bluemke DA, Zerhouni EA. MRI of avascular necrosis of bone. Top Magn Reson Imaging. 1996;8(4):231-46.

18. Miller IL, Savory CG, Polly Jr DW, Graham GD, McCabe JM, Callaghan JJ. Femoral head osteonecrosis. Detection by magnetic resonance imaging versus single-photon emission computed tomography. Clin Orthop Relat Res. 1989;247:152-62.

19. Robinson HJ, Hartleben PD, Lund G, Schrieman J. Nuclear magnetic resonance imaging in femoral head osteonecrosis. Bone Circ Bone Necrosis. 1990:277-280.

20. Ryu JS, Kim JS, Moon DH, Kim SM, Shin MJ, Chang JS, et al. Bone SPECT is more sensitive than MRI in the detection of early osteonecrosis of the femoral head after renal transplantation. J Nucl Med. 2002;43(8):1006-11.

21. Lee GC, Khoury V, Steinberg D, Kim W, Dalinka M, Steinberg M. How do radiologists evaluate osteonecrosis? Skeletal Radiol. 2014;43(5):607-14.

22. Plakseychuk AY, Shah M, Varitimidis SE, Rubash HE, Sotereanos D. Classification of osteonecrosis of the femoral head. Reliability, reproducibility, and prognostic value. Clin Orthop Relat Res. 2001;386:34-41.

23. Smith SW, Meyer RA, Connor PM, Smith SE, Hanley Jr EN. Interobserver reliability and intraobserver reproducibility of the modified Ficat classification system of osteonecrosis of the femoral head. J Bone Joint Surg Am. 1996;78(11):1702-6. 
24. Yamamoto T, Yamaguchi T, Lee KB, Bullough PG. A clinicopathologic study of osteonecrosis in the osteoarthritic hip. Osteoarthritis Cartilage. 2000;8(4):303-8.

25. Lee GC, Steinberg ME. Are we evaluating osteonecrosis adequately? Int Orthop. 2012;36(12):2433-9.
26. Yamamoto T, Schneider R, Iwamoto Y, Bullough PG. Bilateral rapidly destructive arthrosis of the hip joint resulting from subchondral fracture with superimposed secondary osteonecrosis. Skeletal Radiol. 2009;39(2):189-92. 